\title{
FUNDAMENTALS OF COAXIAL AND COPLANAR COIL ARRAYS IN INDUCTION TOOLS
}

\author{
Paulo Roberto de Carvalho ${ }^{1,2}$, Walleson Gomes dos Santos ${ }^{2,3}$ and Cícero Roberto Teixeira Régis ${ }^{2,3}$ \\ Recebido em 18 maio, 2009 / Aceito em 19 fevereiro, 2010 \\ Received on May 18, 2009 / Accepted on February 19, 2010
}

\begin{abstract}
Until about ten years ago, all commercial borehole induction devices were built up with the traditional coaxial coil arrays. Since then, those apparatus incorporated the unconventional coplanar coil arrays, in order to investigate thinly laminated reservoirs and to locate axially asymmetrical anomalies like vugs and fractures. In order to comprehend the fundamentals of the coplanar array in induction probes, we present a comparative study with the coaxial array through onedimensional modeling of some borehole environments, like: 1) homogeneous, isotropic and unlimited conducting media; 2) invaded thick bed with annulus; 3) horizontal and dipping multilayer sequences; 4) gradational transition zone between two thick beds; and 5) thinly laminated formations. The comparative study allows us to conclude that: 1) the skin effect is strongest in the coplanar array responses; 2) the coplanar array responses are more sensitive to mud filtrate invasion and annulus zones, which are direct indicators of movable oil; 3) polarization "horns" on the coplanar array profiles can be good bed boundary indicators; 4) coplanar arrays present the greatest sensitivity to detect and delineate thinly laminated reservoirs.
\end{abstract}

Keywords: well logging, induction tools, thinly reservoirs, coplanar array.

RESUMO. Por meio século, dede sua criação, todas as sondas comerciais por indução eletromagnética (EM) utilizavam o tradicional arranjo coaxial de bobinas. Somente a partir da virada do século XX que estas sondas passaram a incorporar o arranjo coplanar, devido à necessidade de investigar reservatórios finamente laminados ou anomalias sem simetrias de rotação (fraturas ou cavidades). Com o objetivo de melhor compreender a aplicação do arranjo coplanar nas sondas de indução, elaboramos um estudo comparativo de suas respostas com as do tradicional arranjo coaxial, através de modelagem unidimensional, em alguns ambientes comuns à geofísica de poço: 1) meios homogêneos, isotrópicos e ilimitados; 2) camadas espessas com invasão de filtrado de lama e formação de annu/us, 3) sequências de multicamadas horizontais e inclinadas; 4) transição gradacional entre duas camadas espessas; e 5) formações finamente laminadas. Este estudo comparativo entre os arranjos coaxial e coplanar permite concluir que: 1) o efeito pelicular é mais acentuado nas respostas do arranjo coplanar; 2) as respostas do arranjo coplanar são mais sensíveis a movimentação de fluidos na formação, principalmente nas zonas de annulus, 3) os perfis do arranjo coplanar apresentam picos devidos à polarização nas interfaces, que podem ser bons indicadores das fronteiras entre camadas; 4) 0 arranjo coplanar é mais sensível para detectar e delinear reservatórios finamente laminados.

Palavras-chave: perfilagem em poço, ferramentas de indução, reservatório laminado, arranjo coplanar.

\footnotetext{
${ }^{1}$ Instituto Ciberespacial - ICIBE, Universidade Federal Rural da Amazônia - UFRA, Av. Presidente Tancredo Neves, 2501, Terra Firme, 66077-530 Belém, PA, Brazil. Phone: +55(91) 3210-5109 - E-mail: paulo.carvalho@ufra.edu.br

2 Programa de Pós-graduação em Geofísica - CPGf, Universidade Federal do Pará - UFPA, Av. Augusto Corrêa, 01, Guamá, 66075-110 Belém, PA, Brazil. Phone: +55(91) 3201-7692; Phone/Fax: +55(91) 3201-7693 -E-mails: walleson_santos@yahoo.com.br; cicero@ufpa.br

3 Instituto Nacional de Ciência e Tecnologia de Geofísica do Petróleo - INCT-GP.
} 


\section{INTRODUCTION}

None of the traditional E.M. commercial borehole induction devices possessed azimuthal focusing properties until about 10 years ago, whereas the unconventional coplanar coil array had, by design, a strong azimuthal focus. This characteristic of coplanar coil arrays had been explored for many decades in surface electromagnetic surveys. That prompted Moran \& Gianzero (1979) and Kaufman \& Keller (1989) to investigate the application of this transverse EM induction array in simple geometries of the borehole environments. For a better understanding of the coplanar responses in a borehole, the group led by Prof. Om Verma in the Federal University of Pará build laboratory models (Carvalho \& Verma, 1994; Souza \& Verma, 1995; Carvalho \& Verma, 1998) as well as one-dimensional numerical models (Carvalho, 2000; Santos, 2007). Kriegshäuser et al. (2000) presented a multi-coil array to evaluate thinly laminated sand-shale sequences, encountered in deep-water turbidites. Basically, in such triaxial induction tools there are three source coils, one that is coaxial with the borehole axis and two that are transverse to it as shown in Figure 1. Wang et al. (2003) showed that the nine magnetic field components are different only in tridimensional and/or anisotropic environments. Lu \& Alumbaugh (2001) applied the six cross-coupled components to define the tool's azimuthal position into the borehole. Souza \& Verma (1995) were one of the first to explore the azimuthal focusing properties of the coplanar array in the borehole investigation of asymmetrical geological situations such as vugular and fracture zones.

Two major limitations of uniaxial induction tools (coaxial arrays) are the incorrect resistivity reading in dipping beds and in anisotropic layers. Anderson et al. (2008) show some case studies where these limitations are overcome by the triaxial induction (coaxial and coplanar arrays together) measurements. More accurate resistivity leads to more accurate water saturation, which enables petrophysicists to correctly evaluate hydrocarbon reservoirs.

As Ellis \& Singer (2007), in this work we ignore the six crosscoupled components and compare only the responses obtained from the coaxial coil array with that of the coplanar coil arrays, inasmuch as they are the most important signals of the modern induction logging tools. This leaves us with only three different components of the magnetic field.

In order to obtain results of some generality while maintaining a degree of simplicity in the modeling, the results presented here are limited to the basic two-coil arrays. In our theoretical treatment, the coils are represented as point magnetic dipoles since the radii of real coils in induction tools are considered very small compared to the coil spacing and the wavelength (Santos, 2007). Howard (1997) shows that the loop with axis parallel to planar interface and the HMD model responses can depart by 10 to $15 \%$ when the coil spacing is less than four times the loop radii. Therefore, in our models, the sources may be considered magnetic dipoles (VMD and HMD), since their coil spacings are greater than ten times the loop radii.

\section{Homogeneous medium}

A first step in our study is to calculate the responses of the transmitter-receiver arrays in an infinite homogeneous conducting medium. This simple case yields some qualitative ideas on skin effects in induction logging for both coil arrays.

For sinusoidally time varying fields as $e^{i w t}$, where $i=$ $\sqrt{-1}$ and $\omega$ is the angular frequency, the voltages induced in the receivers for both coil arrays, in the frequency domain, are $V=-i \omega \mu n_{r} H \pi a^{2}$, where $\mu$ is the magnetic permeability, $n_{r}$ the number of turns in the receiver coil, $H$ the magnetic field component normal to the plane of the coil of radius $a$. Dividing this voltage by the coaxial and coplanar apparatus constant and expanding in powers of $L / \delta$, where $L$ is the transmitter-toreceiver spacing and $\delta$ the skin depth defined as $\delta=\sqrt{2 / \omega \mu \sigma}$, yields the components of the complex conductivity signal for coaxial, Eq. (1) (Anderson \& Barber, 1997), and coplanar, Eq. (2) (Carvalho \& Verma, 1999), coil arrays:

$$
\begin{gathered}
\sigma_{R}^{c x}+i \sigma_{X F}^{c x}= \\
\sigma-\frac{2 i}{\omega \mu L^{2}}-\frac{2}{3}\left(\frac{L}{\delta}\right) \sigma(1+i)+\cdots ; \\
\sigma_{R}^{c p}+i \sigma_{X F}^{c p}= \\
\frac{2 i}{\omega \mu L^{2}}-\frac{4}{3}\left(\frac{L}{\delta}\right) \sigma(1+i)+\cdots
\end{gathered}
$$

The first terms in the right side of these equations are those obtained by geometrical factor theory, the second terms represent the mutual inductance between the transmitter and the receiver coils in air and the third onward are the conductivity dependent skin effect terms which are ignored in the geometric factor theory. Therefore, the third term provides a first order approximation of the skin effects.

Figure 2 shows the coaxial and coplanar complex conductivities versus the true conductivities of the medium $\left(\sigma_{t}\right)$ through frequency investigation ((a) and (b)) with coil spacing $L=1 \mathrm{~m}$ and spatial investigation ((c) and (d)) with frequency $f=20 \mathrm{kHz}$. 
The in-phase response of the coplanar array deviates from linearity much earlier (near $\sigma_{t}=10^{2} \mathrm{mS} / \mathrm{m}$ ) than that of the coaxial array (near $\sigma_{t}=10^{3} \mathrm{mS} / \mathrm{m}$ ) for all frequencies and coil distances studied.

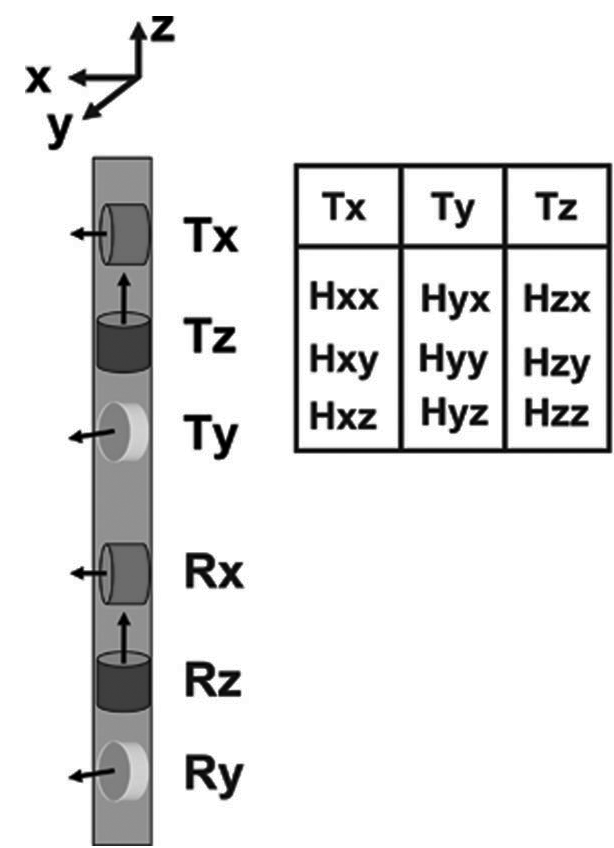

Figure 1 - Three-array (triaxial) induction tool and their nine magnetic field components.

Eq. (3) (Anderson \& Barber, 1997) and Eq. (4) (Carvalho, $2000)$ yield the corrected conductivity for coaxial $\left(\sigma_{c}^{c x}\right)$ and coplanar $\left(\sigma_{c}^{c p}\right)$ coil array, respectively:

$$
\begin{aligned}
& \sigma_{c}^{c x}=\frac{\sigma_{R}^{c x}}{[1-(2 / 3)(L / \delta)]} ; \\
& \sigma_{c}^{c p}=\frac{\sigma_{R}^{c p}}{[1-(4 / 3)(L / \delta)]} .
\end{aligned}
$$

Figure 3 shows the coaxial and coplanar conductivities (resistive, reactive and corrected) in a homogeneous medium in terms of the coil spacing. Solid lines are analytical solutions, circle and star lines are semi-analytical solutions for the cylindrical (Appendix A) and plane parallel boundaries (Appendix B), respectively, without conductivity contrast. Naturally, there is a good convergence among the three ways to generate the same responses. The resistive responses show that the skin effects are more intense in the coplanar than in the coaxial array. However, this loss in the in-phase responses can be counterbalanced by the corresponding gain in its quadrature counterpart for both coil arrays, thus after applying skin effect corrections (SEC) in both systems, the disadvantage between them is fully compensated in homogeneous media.

\section{Nonhomogeneous media}

In our next models we include variations in the conductivity of the media. We accomplish that by simulating two kinds of models that allow us to treat the field propagation as one-dimensional: one formed by coaxial cylindrical shells of infinite length of uniform isotropic electrical conductivity and the other formed by plane parallel layers, each also uniform and isotropic.

Within those models the fields are calculated, in the quasistatic approximation, as a diffusion equation by use of the magnetic an electric scalar potentials, which are associated with the transverse magnetic (TM) and the transverse electric (TE) polarization modes of field propagation, respectively. By applying appropriate boundary conditions to the fields on the interfaces between homogeneous regions the surface impedances and the radial and axial components of the magnetic field are presented in a recursion formulation (Appendixes $A$ and $B$ ) generated for the vertical magnetic dipole (VMD) and the horizontal magnetic dipole (HMD).

\section{Radial investigations}

The radial responses for both coil arrays are modeled for coaxial cylindrical interfaces representing the borehole and the invaded zones in a very thick bed. This approach to invasion profiling assumes that the top and bottom of the bed in which the probe is located are significantly distant from it and, therefore, that radial and vertical responses are separable.

Eqs. (9) and (10) (Kaufman \& Keller, 1989; Carvalho, 2000) yield the apparent conductivities (resistive signals) for coaxial $\left(\sigma_{c}^{c x}\right)$ and coplanar $\left(\sigma_{c}^{c p}\right)$ coil array, respectively:

$$
\begin{aligned}
\sigma_{R}^{c x} & =\frac{2}{\omega \mu L^{2}} Q H_{z} ; \\
\sigma_{R}^{c p} & =\frac{2}{\omega \mu L^{2}} Q H_{r}
\end{aligned}
$$

where $Q H_{z}$ and $Q H_{r}$ are the quadrature of the axial and radial magnetic field components generated by the VMD and the HMD respectively (Appendix A).

An estimate of the influence of the invaded zone is given in Figure 4. This diagram shows the responses of the coaxial (Moran \& Kunz, 1962) and coplanar arrays as a function of the coil spacing. The conductivity of the noninvaded formation is $\sigma_{t}=1,000 \mathrm{mS} / \mathrm{m}$, corresponding to a skin depth of approximately $3.5 \mathrm{~m}$ (140 in). The diameter of invasion is $D_{i}=$ $2.0 \mathrm{~m}$ (80 in). There are three situations plotted in Figure 4: 

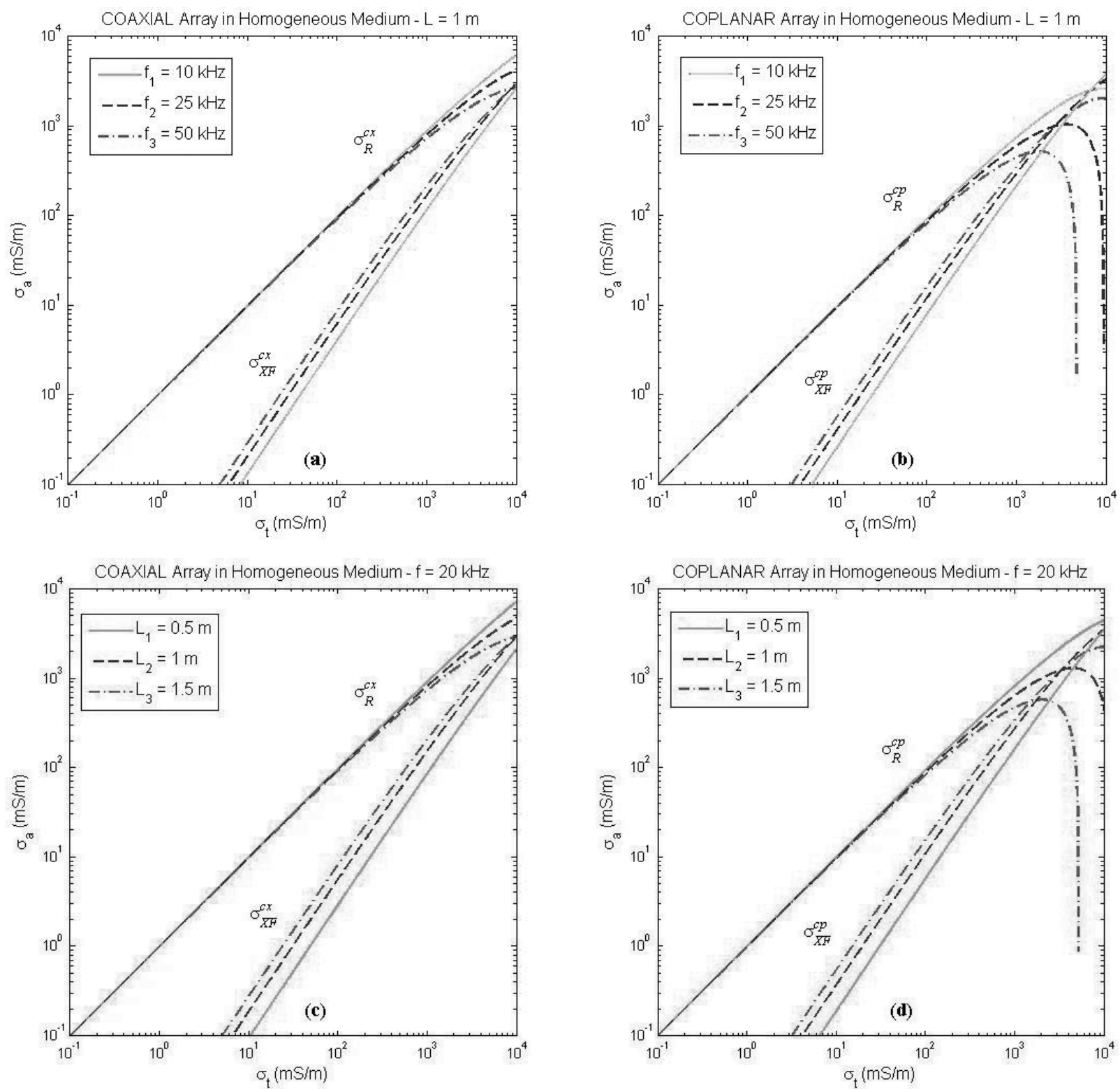

Figure 2 - Resistive $\left(\sigma_{R}^{c x}\right.$ and $\left.\sigma_{R}^{c p}\right)$ and reactive $\left(\sigma_{X P}^{c x}\right.$ and $\left.\sigma_{X P}^{c p}\right)$ responses of the coaxial and coplanar arrays in a homogeneous medium for three different frequencies ((a) and (b)) and three coil spacings ((c) and (d)).

In one of these, the conductivity of the invaded zone is also taken as $\sigma_{i}=1,000 \mathrm{mS} / \mathrm{m}$, corresponding to a homogeneous medium. The other two show invaded zone conductivities of 250 and $62.5 \mathrm{mS} / \mathrm{m}$, respectively.

If the sondes are in a homogeneous medium then, due to the skin effect, their reading will decrease almost linearly with increasing coil spacing $L$, as the resistive signal shown in Figure 3. However, if the invaded zone has a smaller conductivity than the noninvaded zone, there are two opposing tendencies at work as
$L$ increases. First, there are increases in the sonde responses while the geometrical factor is most significant, and then a decrease when the skin effect phenomenon becomes dominant. The net result, as indicated in Figure 4, is that coplanar responses suffer the influence of the invaded zone and skin effects more intensely than the coaxial responses.

Figure 5 shows radial responses $\left(\sigma_{a} / \sigma_{i}\right)$ as a function of invasion diameter $\left(D_{i} / L\right)$ to six different invaded zone contrasts $\left(\sigma_{t} / \sigma_{i}\right)$. The greater skin effect in the coplanar responses (so- 

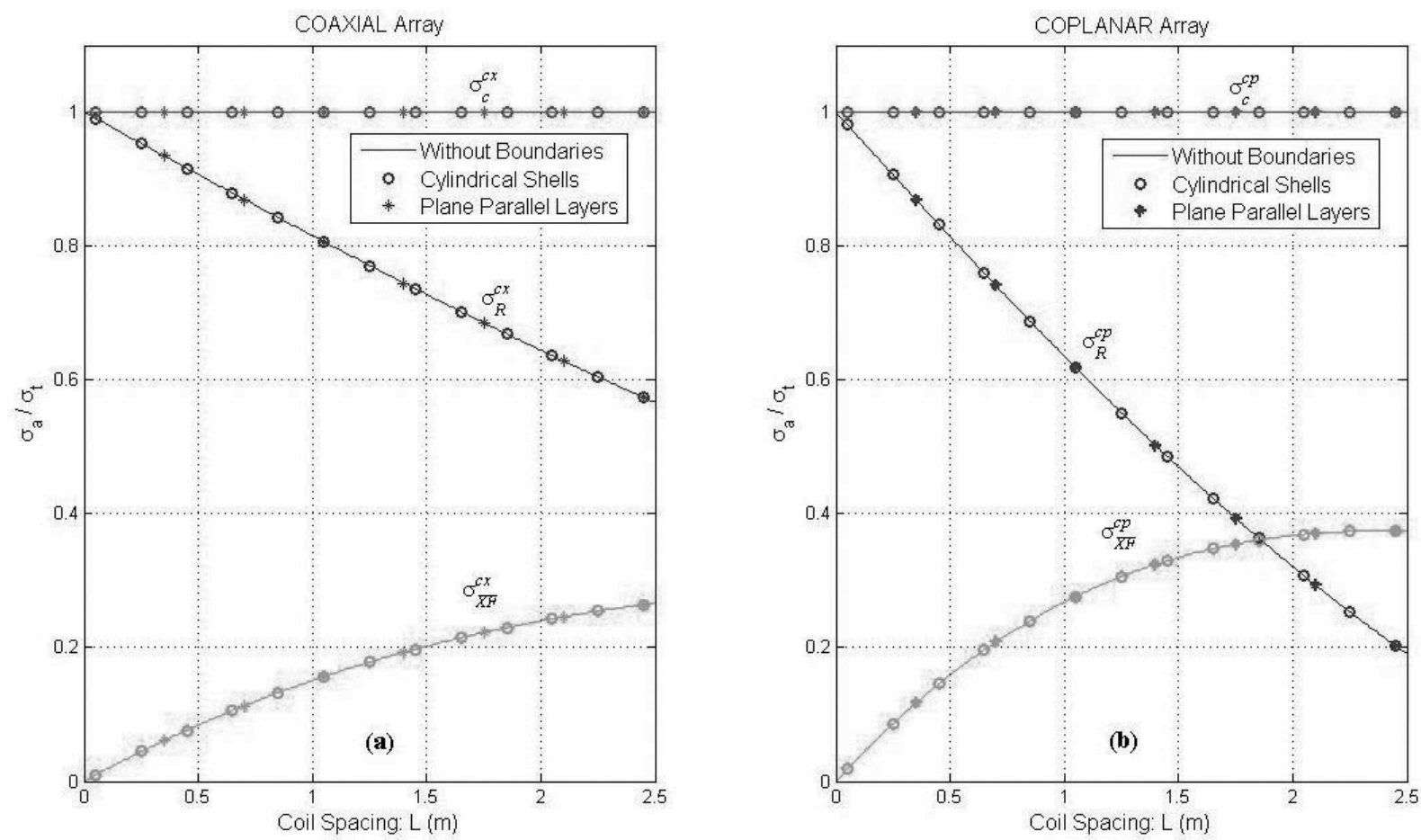

Figure 3 - Resistive $\left(\sigma_{R}^{c x}\right.$ and $\left.\sigma_{R}^{c p}\right)$, reactive $\left(\sigma_{X P}^{c x}\right.$ and $\left.\sigma_{X P}^{c p}\right)$ and corrected $\left(\sigma_{c}^{c x}\right.$ and $\sigma_{C}^{c p}$ ) responses of the (a) coaxial and (b) coplanar arrays in a homogeneous medium versus coil spacing $(L)$. Solid lines are analytical, circle and star lines are semi-analytical solutions for the cylindrical and plane parallel boundaries, respectively, without conductivity contrast.

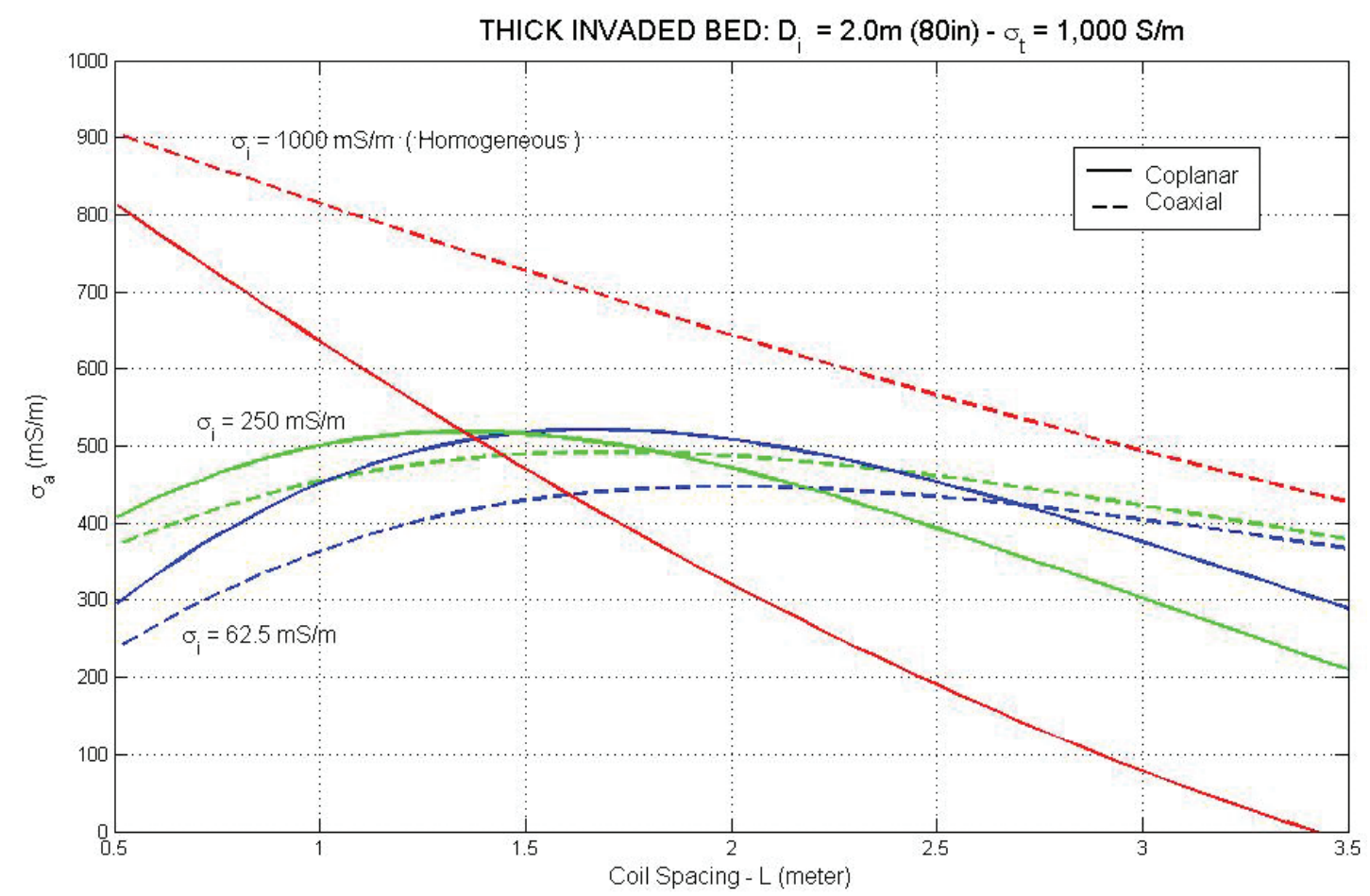

Figure 4 - Apparent conductivity $\left(\sigma_{a}\right)$ versus coil spacing $(L)$, for coplanar (solid lines) and coaxial (dashed lines) two-coils arrays to a thick invaded bed, and for three values of invaded zone conductivity $\left(\sigma_{i}\right)$, with invasion diameter $D_{i}=2.032 \mathrm{~m}$ (80in) and conductivity of noninvaded zone $\sigma_{t}=1 \mathrm{~S} / \mathrm{m}$. 
lid lines) can be seen clearly in the invasion zones with diameter less than $0.2 L$ and invasion contrasts greater than two. For bigger diameter values, the coplanar responses suffer a progressive invasion zone effect due to geometrical factor so that they increase to a maximum. As the invasion diameter increases both coil responses tend to read only the zones disturbed by the mud filtrates $\left(\sigma_{a} \simeq \sigma_{i}\right)$.

Figure 6 shows radial responses $\left(\sigma_{a} / \sigma_{t}\right)$ as a function of coil spacing $\left(L / r_{i}\right)$ to five annulus zone contrasts $\left(\sigma_{a n} / \sigma_{t}\right)$. While the coaxial responses present monotonous deflections, the coplanar responses show greater magnitudes and present oscillations in front of the annulus zones. Under excitation of the field by the HMD the primary vortex electric field, unlike that of a VMD, intersects the boundary between media with different conductivity. For this reason electric charges arise on the interfaces, the density of which changes synchronously with the electric field. Therefore the sources of the total field of the HMD are currents and charges. The annulus is a direct indicator of movable hydrocarbons and this coplanar sensitivity could be explored to position it.

\section{Vertical investigations}

We have studied the coaxial and coplanar profiles in planarparallel interfaces models (Fig. 7) representing the bed boundaries. These models neglect the presence of the borehole and invasion zones.

Eq. (7) (Anderson et al., 1986) and Eq. (8) (Carvalho, 2000) yield the coaxial and coplanar responses, respectively, in terms of the component of the magnetic field, normal to their receivers:

$$
\begin{aligned}
& H_{R}^{c x}=\left[H_{z}^{v}+H_{z}^{h}\right] \cos \theta+\left[H_{r}^{v}+H_{r}^{h}\right] \sin \theta ; \\
& H_{R}^{c p}=\left[H_{z}^{v}+H_{z}^{h}\right] \sin \theta+\left[H_{r}^{h}+H_{r}^{v}\right] \cos \theta
\end{aligned}
$$

where $H^{v}$ and $H^{h}$ are the magnetic fields due to a VMD and $H M D$, respectively (Appendix $B$ ) and $v$ is the angle between the tool axis and the vertical direction.

The first and simplest model chosen to study these responses is formed by two homogeneous half-spaces with a planar horizontal boundary, representing two very thick beds (Fig. 8). We use this model to assess the adjacent bed effect and the skin effect in both coil arrays. We observe that those effects are strongest in the resistive coplanar response. The resistive responses are greater in the coaxial than in the coplanar readings ((a) and (b)), but after the skin effect corrections $((c)$ and $(d))$, both responses approach the true conductivity value of the upper bed $\left(\sigma_{u}\right)$, since it is the background conductivity in Eqs. (3) and (4). When the con- ductivity contrast $\left(\sigma_{u} / \sigma_{d}\right)$ is reduced, the coaxial and coplanar profiles converge to homogeneous media responses, naturally.

Polarization "horns" appear in the coplanar profiles, against the interface. These "horns" are more prominent on resistive logs. They are caused by the building up of the charges at the boundaries, since the normal component of the electric field is discontinuous at the interface. That build-up of charges acts like a secondary transmitter generating a signal in the proximity of the interfaces. Such situation is encountered when the dipoles are not normal to the interfaces like in the cases of a coaxial array in dipping beds or deviated borehole. Anderson et al. (1990) observed that these "horns" don't appear on the field logs of the uniaxial induction tools.

Figure 9 shows a thick $(\mathrm{H}=10 \mathrm{~L})$ conductive horizontal bed $(2 \mathrm{~S} / \mathrm{m})$ in a homogeneous host $(0.5 \mathrm{~S} / \mathrm{m})$. Since the "horns" of the coplanar responses are located directly at the bed boundaries, they may be good quality bed boundary indicators. Kaufman \& Keller (1989) observed that the distance between "horns" is equal to the bed thickness plus the coil spacing. Howard \& Chew (1992) showed theoretically, and Carvalho \& Verma (1994) showed experimentally, through test tank measurements, that these oscillations on the logs are damped if the presence of the borehole and invasion are taken into account.

Figure 10 shows the vertical profiles of the (a) coaxial and (b) coplanar arrays to an inclined bed sequence or a deviated borehole. The vertical profile is the sonde response plotted against its vertical depth. Polarization horns now appear in both profiles against the bed boundaries. These horns are more prominent on resistive logs and in the coplanar profiles they are gradually reduced as the inclination of the well increases. The frequencies affect directly the horn's magnitudes for both coil arrays.

Very frequently the transition between the beds in a geologic sequence is gradational but in the modeling these interfaces are usually represented as sharp boundaries in the form of step profiles. This would cause some discrepancies between the field and synthetic induction borehole profiles. Figure 11 shows the effect of a linear transition zone in the coaxial and coplanar responses. This transition zone was simulated by a sequence of very thin homogeneous layers whose conductivities vary linearly. The main effect of this region over the responses is the smoothing of the "horns" on the coplanar profiles. Anderson et al. (1990) observed that a steep dip angle causes a considerable effect on the induction logs but the main difference between the computed and field logs is the presence of horns on the synthetic logs which do not appear on the field logs. Presently we know that many factors contribute to the appearance of polarization horns such as coil 


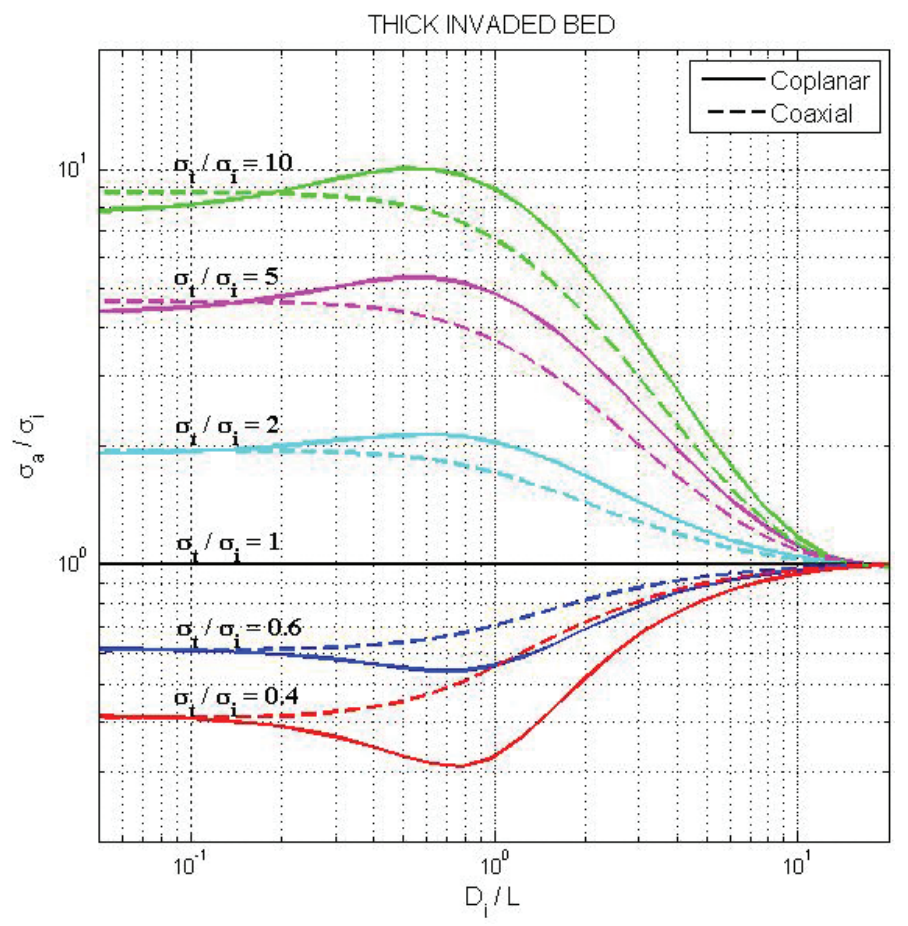

Figure 5 - Coplanar (solid lines) and coaxial (dashed lines) two-coils responses $\left(\sigma_{a} / \sigma_{i}\right)$ in function of the invasion diameter $\left(D_{i} / L\right)$ to a thick invaded bed with six invasion mud filtrates contrast.

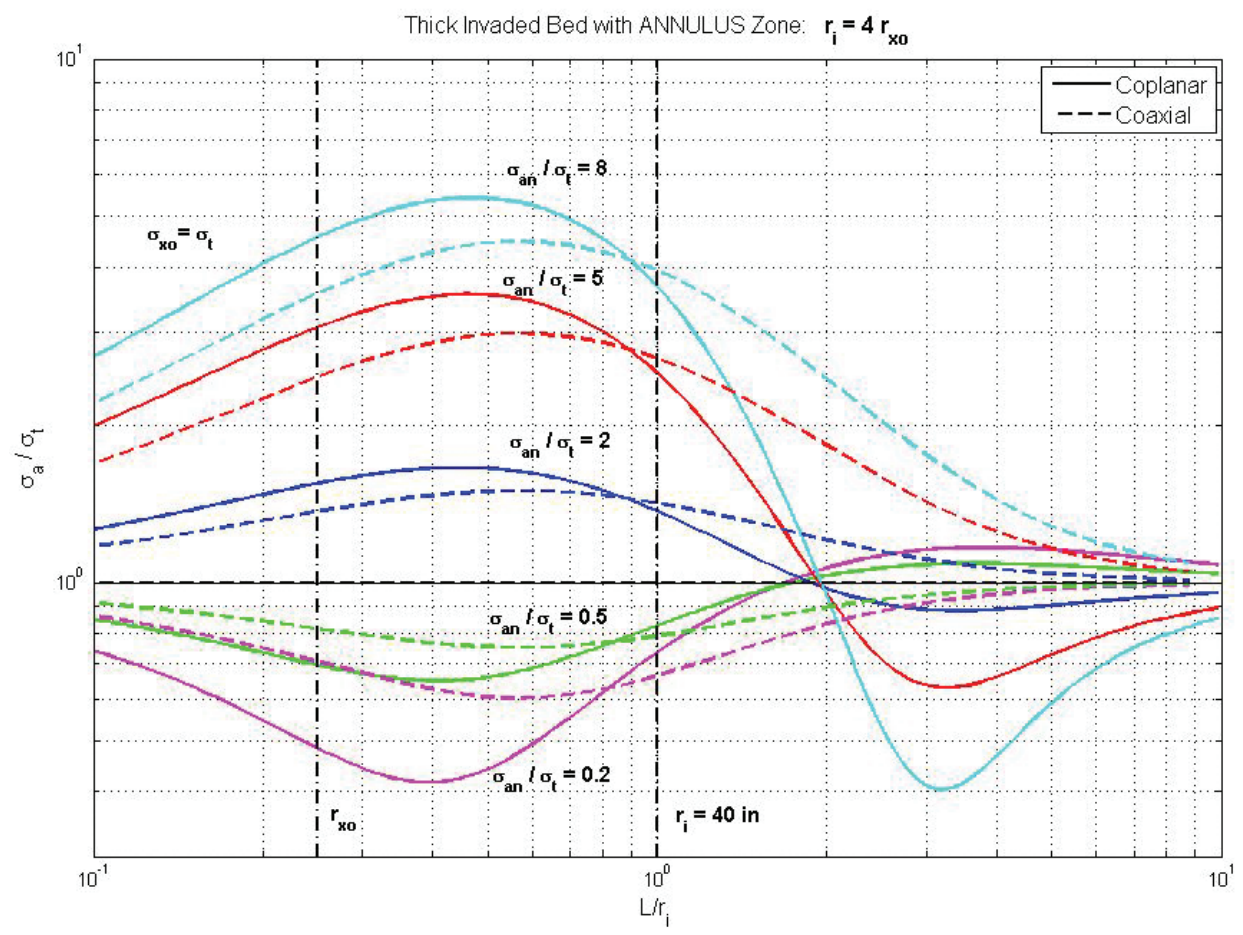

Figure 6 - Coplanar (solid lines) and coaxial (dashed lines) two-coils responses $\left(\sigma_{a} / \sigma_{i}\right)$ in function of the coil spacing $\left(L / r_{i}\right)$ to a thick invaded bed with five kind of annulus zone. 


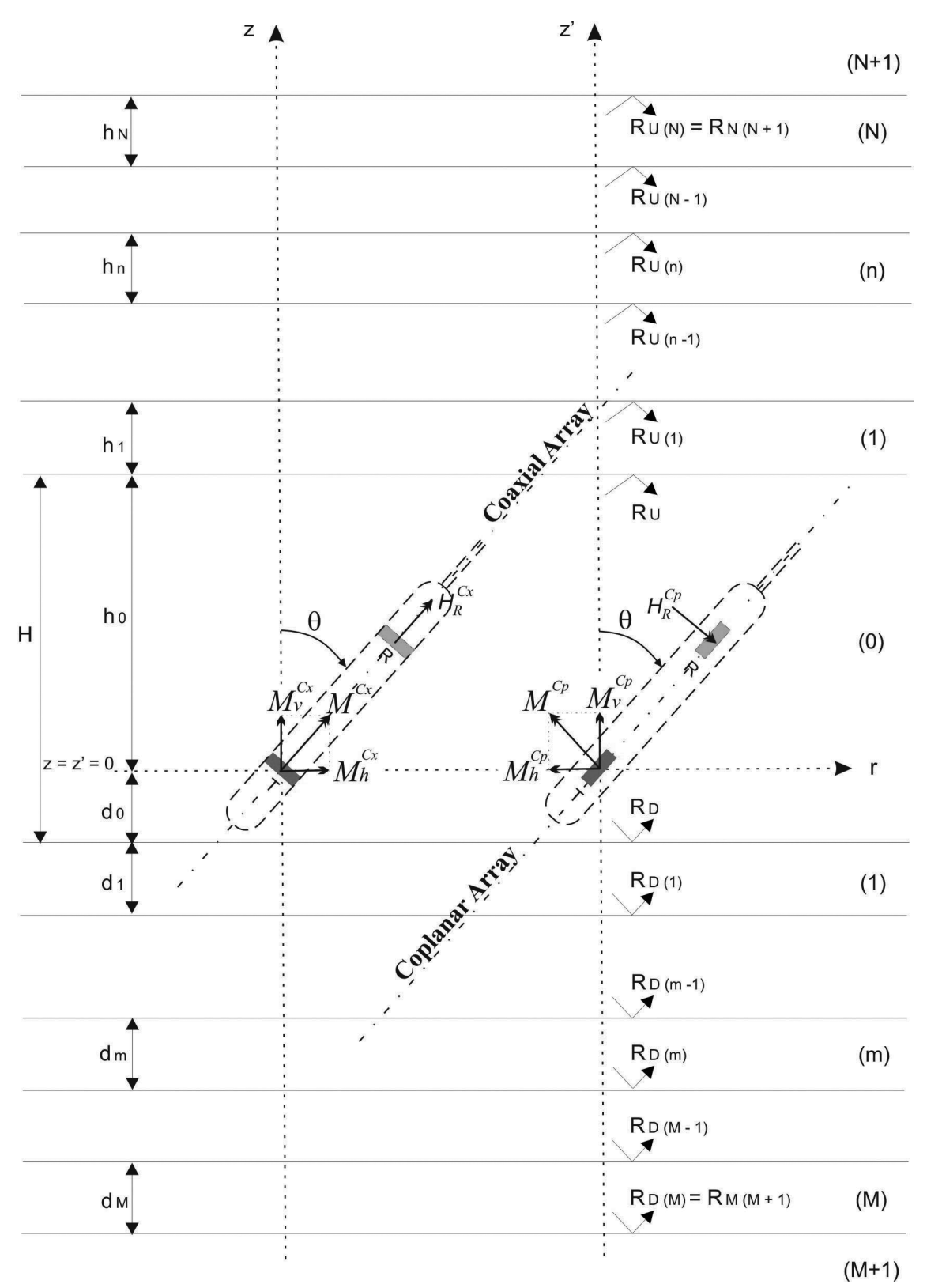

Figure 7 - Schematic representations of coaxial and coplanar two-coils arrays respectively, in a model with planar-parallel interfaces.

size, borehole size, mud conductivity, invasion depth, conductivity contrast between beds. The gradational transition zones can be another important factor for the damping of the polarization horns on the field logs. Therefore, one has to be careful, when using synthetic profiles as an aid to the interpretation of actual profiles, in simply applying "polarization horns" as bed boundary indicators.

Thinly laminated sand-shale sequences, typically encountered in deep-water turbidites, exhibit conductivity anisotropy, i.e., the conductivity varies with direction. Such environments are often mistaken as water-bearing sands because their responses are very similar. Anderson et al. (2008) show a deepwater example of a thin sand-shale turbidite sequence in Krishna-Godavari, off the coast of India, where the thicknesses of the laminae are in millimeter range. Calculated reserves values were $55.5 \%$ higher than those previously obtained using traditional logs and petrophysical evaluation programs.

Figure 12a shows a schematic representation of the induced 

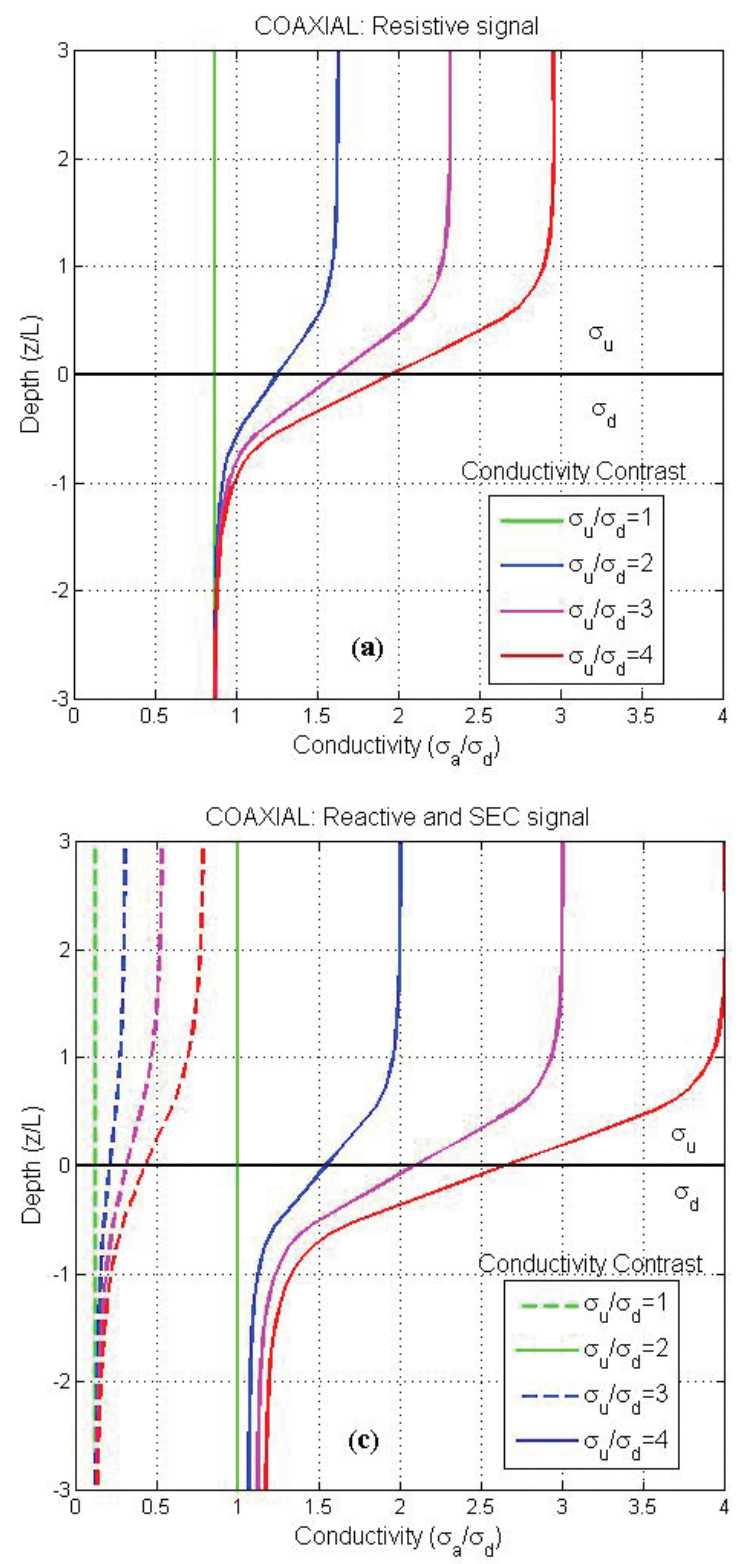
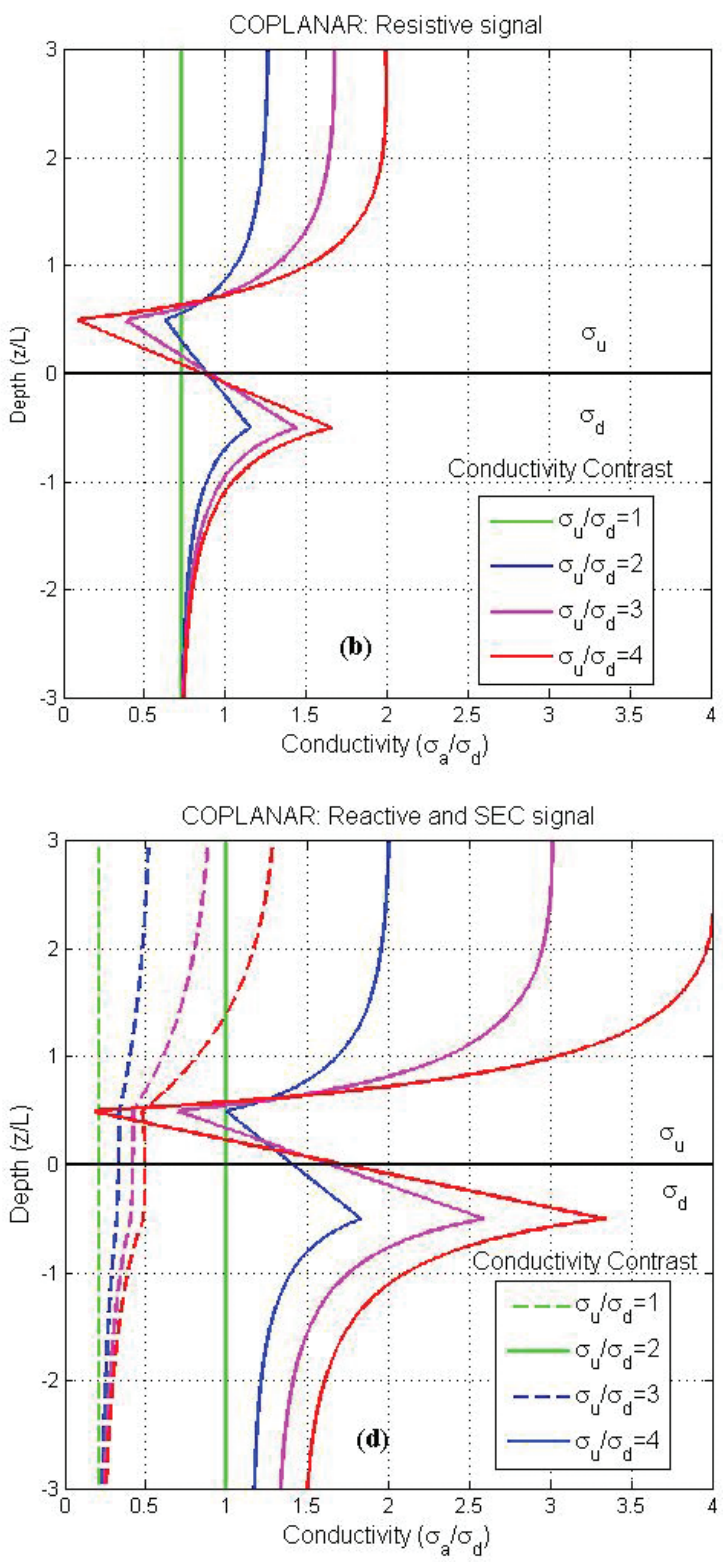

Figure 8 - Resistive, reactive and corrected vertical profiles of the coaxial ((a) and (b)) and coplanar ((c) and (d)) arrays respectively, to a model of a boundary between two very thick beds.

current of a coaxial array in a well that was drilled perpendicular to the bedding in a such formation. This profile will be biased towards the high conductivity laminations (no oil-shale or gasshale) because the induced currents flow mainly parallel to the bedding planes. However, induced currents from a coplanar array (Fig. 12b) will flow perpendicular to the bed interfaces, so that polarization charges will accumulate at layer boundaries. Figure 13 shows the coaxial (a) and coplanar (b) responses in a formation with low contrast where each lamination thickness is $L / 4$. We can see a more prominent oscillation on the coplanar profiles and the "polarization horns" appear again as package boundary indicators.

From circuit theory, the Iongitudinal $\left(\sigma_{h}\right)$ and transverse $\left(\sigma_{v}\right)$ apparent conductivities of the laminated formation (Anderson et al., 1990; Santos, 2007) are obtained by:

$$
\begin{aligned}
& \sigma_{h}=\sigma_{f} V_{f}+\sigma_{a} V_{a} ; \\
& \sigma_{v}=\left(V_{f} / \sigma_{f}+V_{a} / \sigma_{a}\right)^{-1}
\end{aligned}
$$



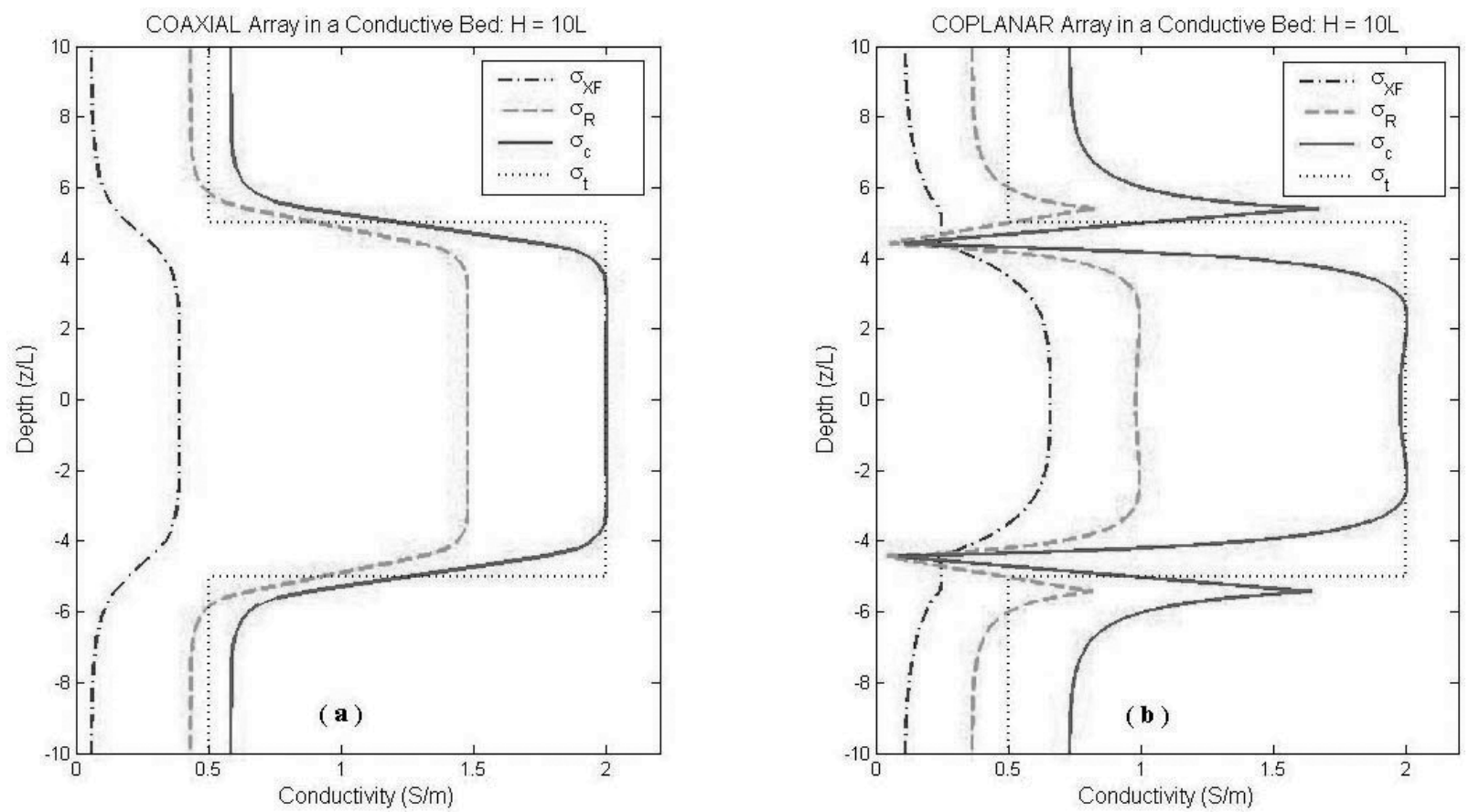

Figure 9 - Resistive $\left(\sigma_{R}\right)$, reactive ( $\sigma_{X F}$ ) and corrected $\left(\sigma_{c}\right)$ vertical profiles of the coaxial (a) and coplanar (b) arrays respectively, to a model of a conductive bed $\left(\sigma_{t}=2 \mathrm{~S} / \mathrm{m}\right)$ surrounding by two very thick layers $\left(\sigma_{t}=0.5 \mathrm{~S} / \mathrm{m}\right)$.
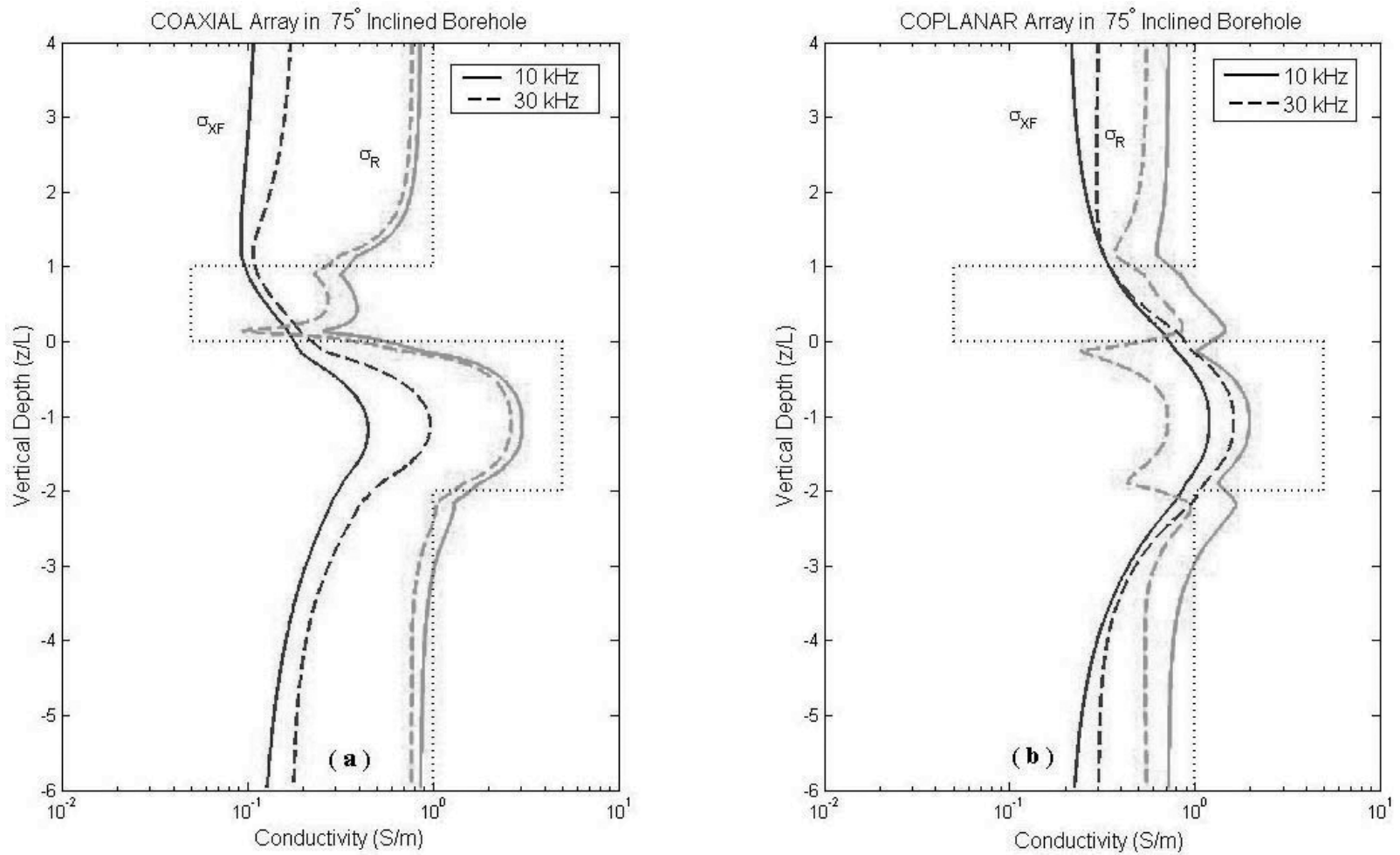

Figure 10 - Resistive $\left(\sigma_{R}\right)$ and reactive ( $\sigma_{X F}$ ) profiles of the coaxial (a) and coplanar (b) arrays respectively, in a $75^{\circ}$ inclined borehole, at $10 \mathrm{kHz}$ (solid lines) and $30 \mathrm{kHz}$ (dashed lines), to adjacent conductive and resistive beds. 

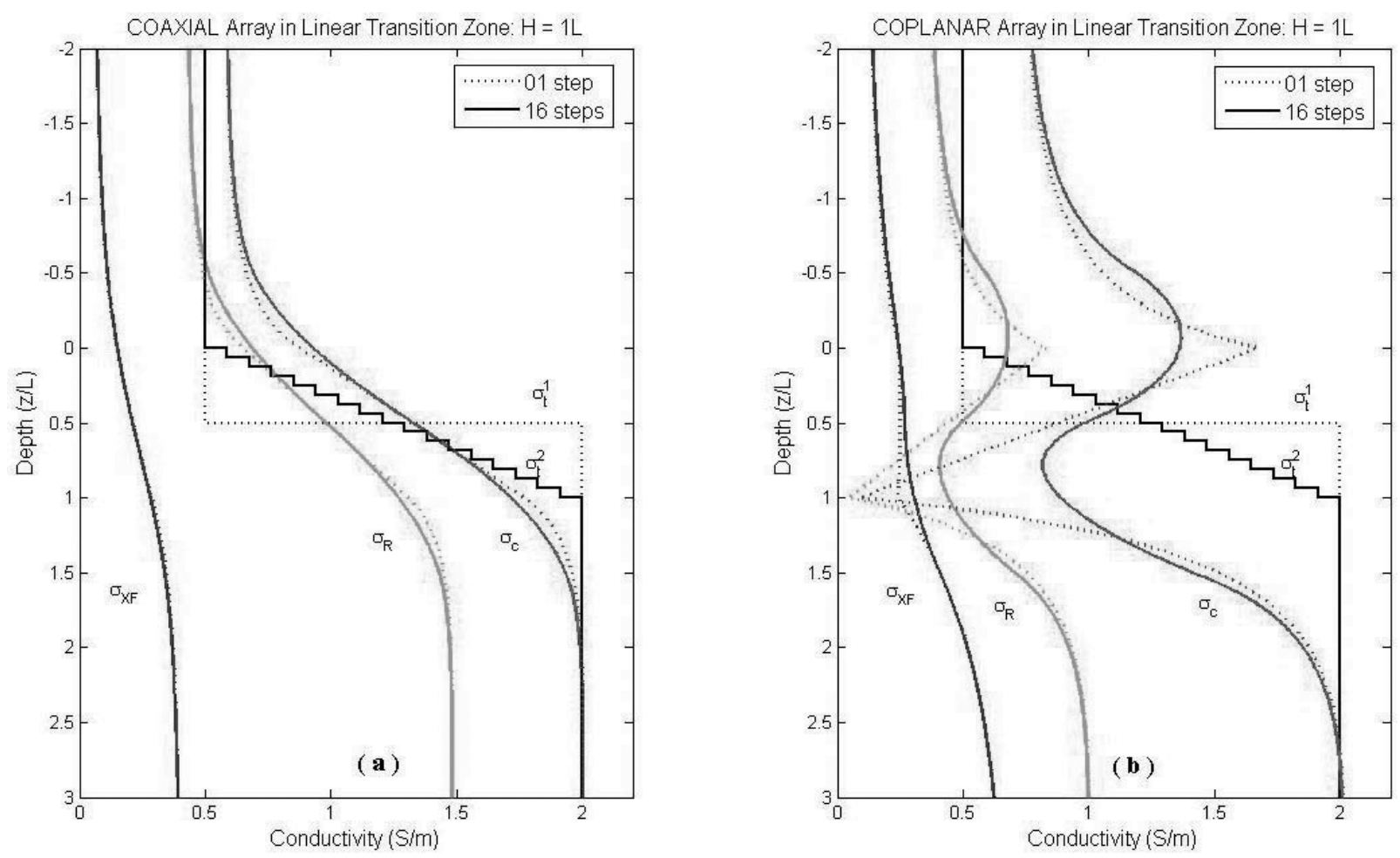

Figure 11 - Resistive $\left(\sigma_{R}\right)$, reactive $\left(\sigma_{X F}\right)$ and corrected $\left(\sigma_{c}\right)$ vertical profiles of the coaxial (a) and coplanar (b) arrays respectively, to a model of step ( $\left.\sigma_{t}^{1}\right)$ and gradational $\left(\sigma_{t}^{2}\right)$ transition between two very thick beds.
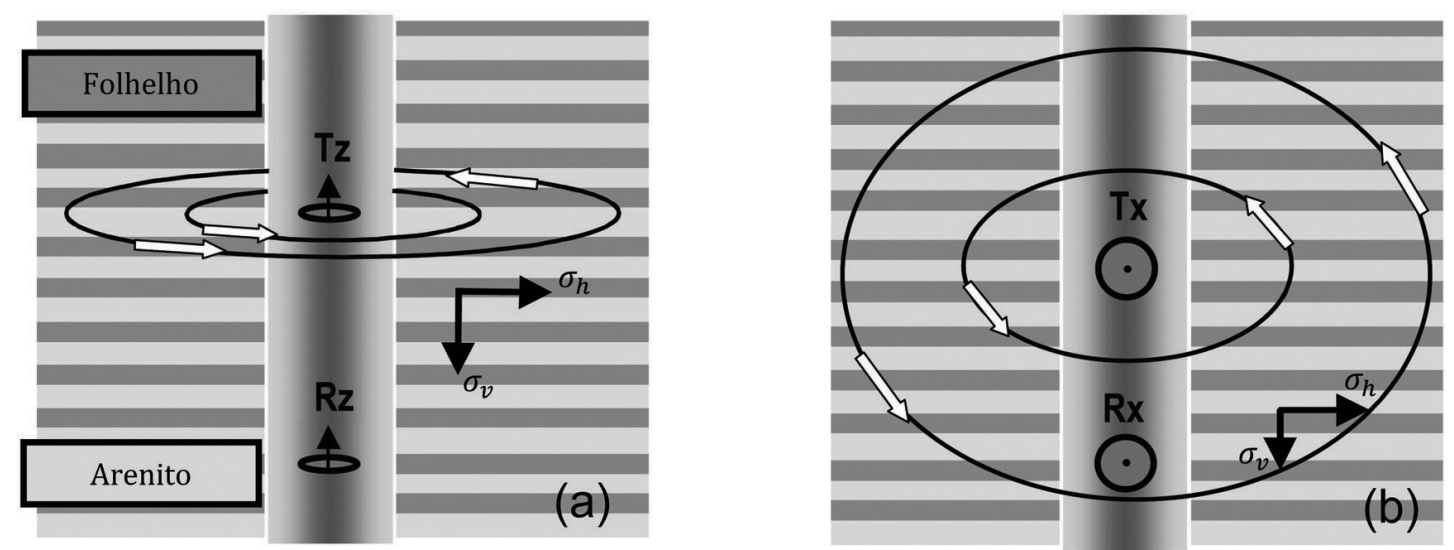

Figure 12 - Schematics representations of the eddy currents generated by coaxial (a) and coplanar (b) arrays respectively, to a model of thinly laminated sequence.

where $\sigma_{f}$ and $\sigma_{a}$ are the shale and sand conductivities; $V_{f}$ and $V_{a}$ are the shale and sand volume in percentage, respectively.

Howard (2000) derives vertical $\left(R_{v}\right)$ and horizontal resistivities in laminated sand-shale sequences defined by relative sandshale volumes and relates it to the apparent resistivity $\left(R_{a}\right)$ as a function of the anisotropic index $\left(\sigma_{h} / \sigma_{v}\right)$ and the dip angle. Anderson et al. (2008) show this anisotropic index is a useful measurement for determining the level of anisotropy, and when this ratio is higher than five, it alerts the log analyst to look for potential laminated-pay reservoirs.

Figure 14 shows the three-array's responses ( $L=1 \mathrm{~m} ; f=$ $20 \mathrm{kHz}$ and axis' tool in XZ plane) to a vertical (a) and a $30^{\circ}$ inclined (b) borehole in a thinly laminated $(L / 32)$ sand-shale reservoir sandwiched by a homogeneous host. Eqs. (9) and (10) yield 

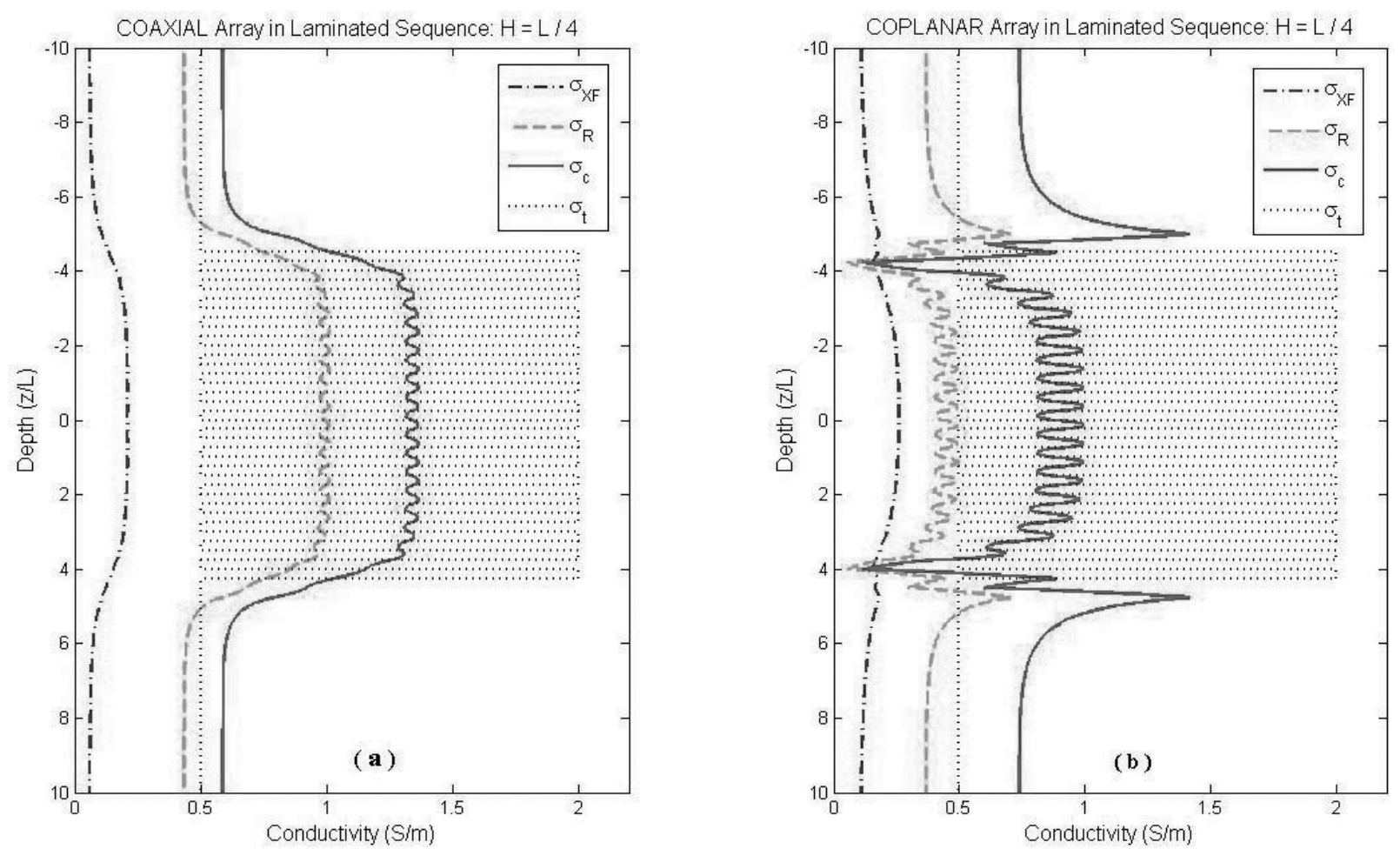

Figure 13 - Resistive $\left(\sigma_{R}\right)$, reactive $\left(\sigma_{X F}\right)$ and corrected $\left(\sigma_{c}\right)$ vertical profiles of the coaxial (a) and coplanar (b) arrays respectively, to a model of $\left(\sigma_{t}\right)$ laminated ( $L / 4)$ sequence.

the transverse $\left(\sigma_{h}=0.18 \mathrm{~S} / \mathrm{m}\right)$ and vertical $\left(\sigma_{v}=0.55 \mathrm{~S} / \mathrm{m}\right)$ conductivities, respectively. In both situations the coaxial array's responses $\left(\sigma_{z z}\right)$ are more influenced by the conductive laminas than the coplanar responses $\left(\sigma_{x x}\right.$ and $\left.\sigma_{y y}\right)$ since in the first case the currents are induced in horizontal planes following the conductive layers. When the borehole is tilted, the dipole source in the coaxial array is no longer perpendicular to the interfaces and its response starts to be influenced by the vertical conductivity. The opposite effect occurs with the coplanar array's responses, which are then influenced by the horizontal conductivity. In this case, not only the measured conductivity increases, but also the $\sigma_{x x}$ and $\sigma_{y y}$ show two different curves, because of the lack of symmetry.

\section{CONCLUSIONS}

Many important sand-shale reservoirs were often mistaken as water-bearing sands until ten years ago because the induction logs were obtained with only the traditional coaxial coil array. Under excitation by the coplanar coil array, the primary vortex electric field, unlike that of a coaxial array, intersects the boundary between media with different conductivity. For this reason electric charges arise on the interfaces, the density of which changes synchronously with the electric field. Therefore the sources of the total field of the coplanar are currents and surface charges. Another important property of the coplanar array that was neglected for a long time is its azimuthal focusing that can be explored in axially asymmetrical situations such as vugs, fracture zones and invasion zones in horizontal wells.

The components of the electric field normal to the interfaces between different media and the resulting surface charge build up are responsible for the most useful and unique features of the coplanar array responses, like the ones listed below.

Based on the comparative study between the coaxial and the coplanar coil arrays we conclude that:

1. the skin effects are strongest in the coplanar responses, but this disadvantage is partially compensated by applying the corrections for these effects;

2. the coplanar response is more sensitive to mud filtrate invasion and annulus zones, which can be direct indicators of movable hydrocarbons;

3. the polarization "horns" are obtained in the coplanar pro- 

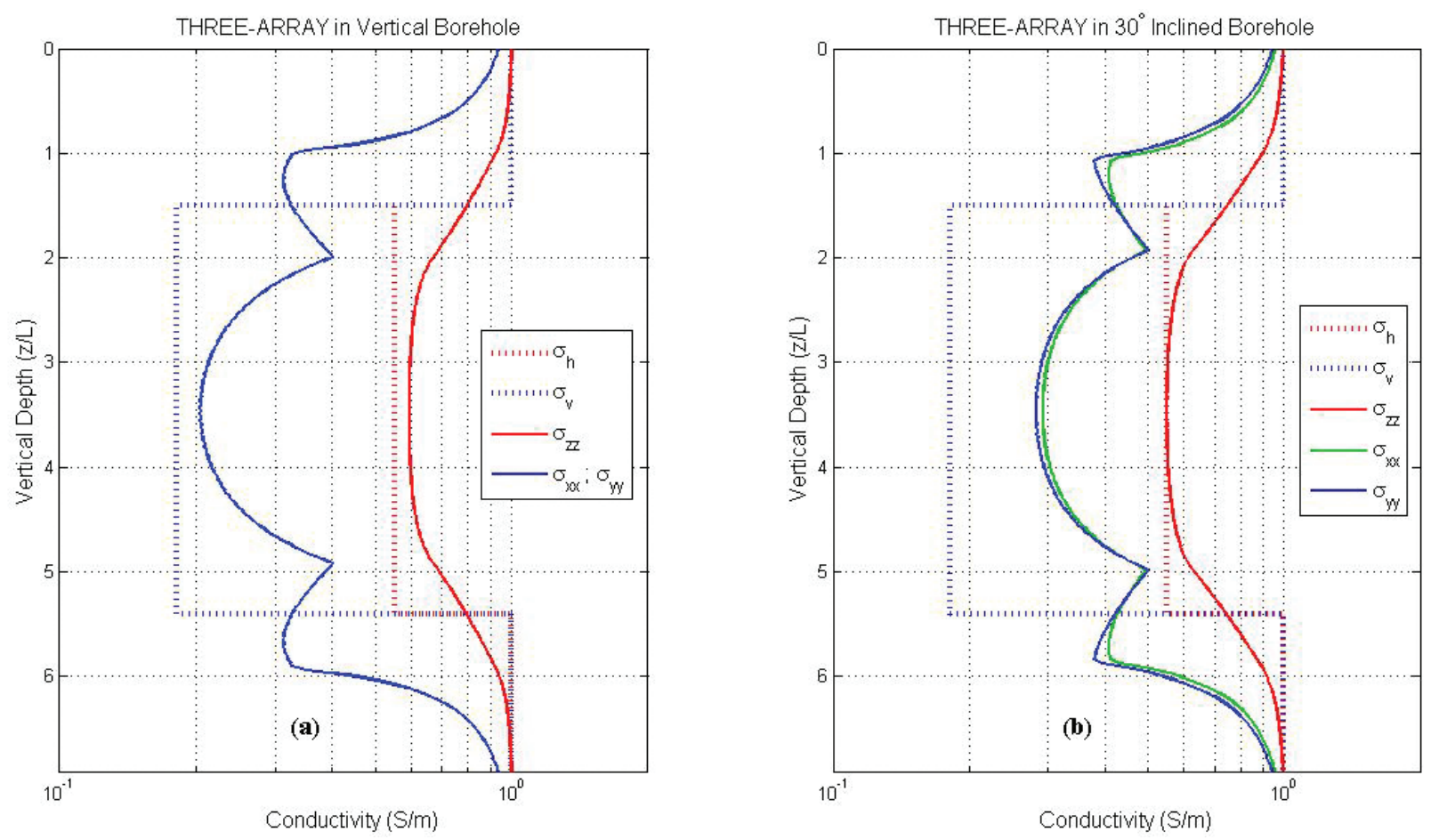

Figure 14 - Three-array's responses in a vertical borehole (a) and in $30^{\circ}$ inclined borehole (b) to a thinly laminated $(L / 32)$ sand $(0.1 \mathrm{~S} / \mathrm{m}) / \mathrm{shale}(1 \mathrm{~S} / \mathrm{m})$ sequence $(4 L)$.

files in front of bed boundaries, consequently, they can be indicators of interface positions;

4. the coplanar response is more sensitive to detect and delineate thinly laminated reservoirs.

\section{ACKNOWLEDGMENTS}

Many have published their work on induction logging, but there are many (mainly physical scale modeling) who may not be as well recognized. Among these who deserve recognition is Prof. Dr. Om Prakash Verma, who took the Doll and Kaufman' ideas and made them work to extend the range of applicability of the induction logging, back in the 1990's. This paper is dedicated to him in loving memory.

\section{APPENDIX A - Coaxial-cylindrical interfaces}

The axial $\left(H_{z}\right)$ and radial $\left(H_{r}\right)$ components of magnetic field in cylindrical coordinate system, generated for the vertical magnetic dipole (VMD) and horizontal magnetic dipole (HMD), normal to receiver coils of the coaxial and coplanar sondes respectively, are presented in recursion formulation in a model formed by coaxialcylindrical boundaries between homogeneous regions (Fig. 4).
The electric and magnetic fields due to the sources (VMD and HMD) may be obtained in terms of electric $\vec{F}$ and magnetic $\vec{A}$ vectors potentials (Ward \& Hohmann, 1988) by Eqs. (A-1) and $(\mathrm{A}-2)$ :

$$
\begin{aligned}
\vec{E} & =-\hat{z} \vec{A}+\nabla(\nabla \cdot \vec{A}) / \hat{y}-\nabla \times \vec{F} ; \\
\vec{H} & =-\hat{y} \vec{F}+\nabla(\nabla \cdot \vec{F}) / \hat{z}-\nabla \times \vec{A}
\end{aligned}
$$

where $\hat{z}$ and $\hat{y}$ are the impeditivity (ohm/m) and admittivity (S/m) of the media, respectively. $\vec{F}$ and $\vec{A}$ are obtained by solutions of the sets:

$$
\begin{aligned}
\nabla^{2} \vec{F}+k^{2} \vec{F} & =-\hat{z} \vec{m} \delta(x) \delta(y) \delta(z) ; \\
\nabla^{2} \vec{A}+k^{2} \vec{A} & =0
\end{aligned}
$$

where $\vec{m}$ is the magnetic dipole moment.

As the sources should be oriented like $\vec{m}=\left(0,0, m_{z}\right)$ for the VMD, or $\vec{m}=\left(m_{r}, 0,0\right)$ for the HMD, the vector potentials have a single component each: $\vec{F}=\left(F_{r}, 0,0\right)$ and $\vec{A}\left(A_{r}, 0,0\right)$. For a convenient notation, they may be treated like scalar potentials: $F_{r}=\psi$ and $A_{r}=\theta$. So, the sets of six wave scalar Eqs. (A-3) and (A-4) reduces to only two. 


\section{Vertical Magnetic Dipole - VMD}

The two boundary conditions of the electric $(E)$ and magnetic $(H)$ tangential field components in the interfaces generated by VMD are:

$$
\begin{gathered}
E_{\phi}^{j}=E_{\phi}^{j+1} \Longleftrightarrow \frac{\partial \psi_{j}}{\partial r}=\partial \psi_{j+1} \partial r \\
H_{z}^{j}=H_{z}^{j+1} \Longleftrightarrow k_{j}^{2} \psi_{j}+\frac{\partial^{2} \psi_{j}}{\partial z^{2}} \\
=k_{j+1}^{2} \psi_{j+1}+\frac{\partial^{2} \psi_{j+1}}{\partial z^{2}}
\end{gathered}
$$

where $\phi, r$ and $z$ are the angular, radial and axial of the cylindrical coordinates respectively; $j=1,2, \ldots, n+1$ are the medium and $n$ is the number of interfaces.

The scalar potential $\psi_{j}$ is expressed by

$$
\begin{gathered}
\psi_{j}=\frac{M}{2 \pi^{r}} \int_{0}^{\infty} \\
\times\left[c_{j} I_{0}\left(u_{j} r_{n}\right)+d_{j} K_{0}\left(u_{j} r_{n}\right)\right] \cos \left(k_{r} z\right) d k_{r},
\end{gathered}
$$

where $M$ is the magnetic dipole moment; $I_{0}$ and $K_{0}$ are modified Bessel functions of first and second kind respectively, and order zero; $k_{j}=\sqrt{-i \omega \mu \sigma_{j}}$ and $u_{j}=\sqrt{k_{r}^{2}-k_{j}^{2}}$ are the wavenumber and the wave constant, respectively; $r_{n}$ are the borehole and invasion zones radii. The $2 n$ coefficients in Eq. (A-7) $\left(c_{j}\right.$ and $d_{j}$ ) are obtained by applying $\psi_{j}$ in the two boundary conditions, Eqs. (A-5) and (A-6), and considering two additional conditions: 1) near the source the coefficient $c_{1}$ is equal to unity while in the external medium $d_{n+1}=0$.

The vertical component of magnetic field which is normal to the receiver coil in the coaxial sonde $(r=0$ and $z=L)$ is:

$$
H_{z}=\left(1+i k_{1} L\right) e^{-i k_{1} L}-\left(L^{3} / \pi\right) \int_{0}^{\infty} u_{1}^{2} c_{1} d k_{r} .
$$

\section{Horizontal Magnetic Dipole - HMD}

The four boundary conditions of the electric $(\mathrm{E})$ and magnetic $(\mathrm{H})$ tangential field components in the interfaces generated by HMD are:

$$
\begin{aligned}
E_{z}^{j} & =E_{z}^{j+1} \Longleftrightarrow \frac{1}{\sigma_{j}}\left[k_{j}^{2} \theta_{j}+\frac{\partial^{2} \theta_{j}}{\partial z^{2}}\right] \\
& =\frac{1}{\sigma_{j+1}}\left[k_{j+1}^{2} \theta_{j+1}+\frac{\partial^{2} \theta_{j+1}}{\partial z^{2}}\right],
\end{aligned}
$$

$$
\begin{gathered}
E_{\phi}^{j}=E_{\phi}^{j+1} \Longleftrightarrow \frac{1}{\sigma_{j}}\left[\frac{1}{r_{n}} \frac{\partial^{2} \theta_{j}}{\partial \phi \partial z}-k_{j}^{2} \frac{\partial \psi_{j}}{\partial r}\right] \\
=\frac{1}{\sigma_{j+1}}\left[\frac{1}{r_{n}} \frac{\partial^{2} \theta_{j+1}}{\partial \phi \partial z}-k_{j+1}^{2} \frac{\partial \psi_{j+1}}{\partial r}\right], \\
H_{z}^{j}=H_{z}^{j+1} \Longleftrightarrow k_{j}^{2} \psi_{j}+\frac{\partial^{2} \psi_{j}}{\partial z^{2}} \\
=k_{j+1}^{2} \psi_{j+1}+\frac{\partial^{2} \psi_{j+1}}{\partial z^{2}}, \\
H_{\psi}^{j}=H_{\psi}^{j+1} \Longleftrightarrow-\frac{\partial \theta_{j}}{\partial r}+\frac{1}{r_{n}} \frac{\partial^{2} \psi_{j}}{\partial \phi \partial z} \\
=-\frac{\partial \theta_{j+1}}{\partial r}+\frac{1}{r_{n}} \frac{\partial^{2} \psi_{j+1}}{\partial \phi \partial z} .
\end{gathered}
$$

The potentials $\theta_{j}$ and $\psi+j$ are expressed by

$$
\begin{gathered}
\theta_{j}=\frac{M}{2 \pi^{2}} k_{j}^{2} \sin \phi \int_{0}^{\infty} \\
\times\left[a_{j} K_{1}\left(u_{j} r_{n}\right)+b_{j} I_{1}\left(u_{j} r_{n}\right)\right] \frac{1}{u_{j}} \cos \left(k_{r} z\right) d k_{r}, \\
\psi_{j}=\frac{M}{2 \pi^{2}} k_{j}^{2} \cos \phi \int_{0}^{\infty} \\
\times\left[c_{j} K_{1}\left(u_{j} r_{n}\right)+d_{j} I_{1}\left(u_{j} r_{n}\right)\right] \frac{k_{r}}{u_{j}} \sin \left(k_{r} z\right) d k_{r},
\end{gathered}
$$

where $I_{1}$ and $K_{1}$ are modified Bessel functions of first and second kind respectively and order one. The $4 n$ coefficients in Eqs. (A-13) and (A-14) $\left(a_{j}, b_{j}, c_{j}\right.$ and $\left.d_{j}\right)$ are obtained applying $\theta_{j}$ and $\psi_{j}$ in the four boundary conditions, Eqs. (A-9) to (A-12), and considering two additional conditions: near the source the coefficients $a_{1}$ and $c_{1}$ are equal to unity while in the external medium $b_{n+1}=d_{n+1}=0$.

The radial component of magnetic field which is normal to the receiver coil in the coplanar sonde $(r=0$ and $z=L)$ is:

$$
\begin{aligned}
H_{r}= & {\left[1+i k_{1} L+\left(i k_{1} L\right)^{2}\right] e^{-i k_{1} L}-\left(\alpha^{3} / \pi\right) \int_{0}^{\infty} } \\
& \times\left[k_{1}^{2} r_{1}^{2} b_{1}+m^{2} d_{1}\right] r_{1} \cos \left(L k_{r}\right) d k_{r},
\end{aligned}
$$

where $\alpha=L / r_{1}, m=r_{1} k_{r}$ and $r_{1}$ is the borehole radius. 
These semi-analytical responses were generated by applying routines of the International Mathematical and Statistical Libraries (IMSL) software package. We used the routine QDAWF to solve the numeral improper integrals. According to the package tutorial, this routine uses a globally adaptive scheme in an attempt to reduce the absolute error. Depending on the length of the subinterval in relation to the size of $k_{r}$, either a modified Clenshaw-Curtis procedure or a Gauss-Kronrod rule is employed to approximate the integral on a subinterval.

\section{APPENDIX B - Plane parallel interfaces}

As the sources should be oriented like $\vec{m}=\left(0,0, m_{z}\right)$ for the VMD, or $\vec{m}=\left(m_{r}, 0,0\right)$ for the HMD, the vector po- tentials have a single component each: $\vec{F}=\left(0,0, F_{z}\right)$ and $\vec{A}=\left(0,0, A_{z}\right)$. So, similarly to the cylindrical boundary models (Appendix A), the sets of six scalar wave equations reduce to only two.

The radial and axial components of the magnetic field are presented to a receiver point $z_{n}=L \cos \theta$, generated for the vertical magnetic dipole (VMD) and the horizontal magnetic dipole (HMD) in a model formed by plan-parallel boundaries (Fig. 7). When the receiver is in a different bed than the transmitter, $z_{n}$ becomes the distance between the receiver and the bed boundary on the side of the transmitter.

The reflection coefficients on the interfaces $\left(R_{U_{n}}\right.$ or $\left.R_{D_{m}}\right)$ are obtained applying recursion equations of the form:

$$
\begin{aligned}
& R_{U(N+1)}=R_{D(M+1)}=0 ; \\
& R_{U(n-1)}=\frac{R_{(n-1) n}+R_{U n} e^{i 2 u_{n} h_{n}}}{1+R_{(n-1) n} R_{U n} e^{i 2 u_{n} h_{n}}}
\end{aligned}
$$

and

$$
R_{D(n-1)}=\frac{R_{(n-1) n}+R_{D n} e^{i 2 u_{n} d_{n}}}{1+R_{(n-1) n} R_{D n} e^{i 2 u_{n} d_{n}}}
$$

where $R_{(n-1)_{n}}$ are the reflection coefficient from the ( $\left.n-1\right)$-th layer, known as "medium impedance", and is given by

$$
R_{(n-1) n}=\frac{\alpha_{n} u_{(n-1)}-\alpha_{(n-1)} u_{n}}{\alpha_{n} u_{(n-1)}+\alpha_{(n-1)} u_{n}}
$$

where $\alpha_{n}=\frac{i \sigma_{n}}{\omega}$ for TM and $\alpha_{n}=1$ for TE mode.

\section{Vertical Magnetic Dipole - VMD}

\section{(a) Receiver above the transmitter}

For $z>0$, and in the $\mathrm{n}$-th layer $\left(0<z_{n}<h_{n}, 0<n<N+1\right)$ :

$$
\begin{aligned}
& H_{n z}^{v}=\frac{i M_{v}}{4 \pi} \int_{0}^{\infty}\left[e^{i u_{n} z_{n}}+R_{U n}^{T E} e^{i u_{n}\left(2 h_{n} z_{n}\right)}\right] \frac{1+R_{D}^{T E} e^{i 2 u_{0} d_{0}}}{1+R_{U}^{T E} R_{D}^{T E} e^{i 2 u_{0} H}} A_{n} J_{0}\left(k_{r} r\right) \frac{k_{r}^{3}}{u_{0}} d k_{r}, \\
& H_{n r}^{v}=\frac{M_{v}}{4 \pi} \int_{0}^{\infty}\left[e^{i u_{n} z_{n}}+R_{U n}^{T E} e^{i u_{n}\left(2 h_{n} z_{n}\right)}\right] \frac{1+R_{D}^{T E} e^{i 2 u_{0} d_{0}}}{1-R_{U}^{T E} R_{D}^{T E} e^{i 2 u_{0} H}} A_{n} J_{1}\left(k_{r} r\right) u_{n} \frac{k_{r}^{2}}{u_{0}} d k_{r},
\end{aligned}
$$

where $R_{D}$ and $R_{U}$ are the reflections inside the bed of thickness $H$ in which the source is located; $d_{0}$ is the vertical distance from the source to the interface immediately below it; $h_{n}$ are the thicknesses of the beds above of the source; and are obtained with the recursion relationship,

$$
A_{n}=A_{(n-1)} e^{i u_{(n-1)} h_{(n-1)}} \frac{1+R_{U(n-1)}^{T E}}{1+R_{U n}^{T E} e^{i 2 u_{n} h_{n}}},
$$

with $A_{0}=1$, for the layer where the transmitter is situated. 
(b) Receiver below the transmitter

For $z<0$, and in the n-th layer $\left(-d_{n}<z_{n}<0,0<n<M+1\right)$ :

$$
\begin{gathered}
H_{n z}^{v}=\frac{i M_{v}}{4 \pi} \int_{0}^{\infty}\left[e^{-i u_{n} z_{n}}+R_{D n}^{T E} e^{i u_{n}\left(2 d_{n}+z_{n}\right)}\right] \frac{1+R_{U}^{T E} e^{i 2 u_{0} h_{0}}}{1-R_{U}^{T E} R_{D}^{T E} e^{i 2 u_{0} H}} B_{n} J_{0}\left(k_{r} r\right) \frac{k_{r}^{3}}{u_{0}} d k_{r}, \\
H_{n r}^{v}=-\frac{M_{v}}{4 \pi} \int_{0}^{\infty}\left[e^{-i u_{n} z_{n}}+R_{D n}^{T E} e^{i u_{n}\left(2 d_{n}+z_{n}\right)}\right] \frac{1+R_{D}^{T E} e^{i 2 u_{0} h_{0}}}{1-R_{U}^{T E} R_{D}^{T E} e^{i 2 u_{0} H}} B_{n} J_{1}\left(k_{r} r\right) u_{n} \frac{k_{r}^{2}}{u_{0}} d k_{r},
\end{gathered}
$$

where $h_{0}$ is the vertical distance from the source to the interface immediately above it; $d_{n}$ are the thicknesses of the beds below of the source; and $B_{n}$ are obtained with the recursion relationship,

$$
B_{n}=B_{(n-1)} e^{i u_{(n-1)} d_{(n-1)}} \frac{1+R_{D(n-1)}^{T E}}{1+R_{D n}^{T E} e^{i 2 u_{n} d_{n}}},
$$

with $B_{0}=1$, for the layer where the transmitter is situated.

\section{Horizontal Magnetic Dipole - HMD}

(a) Receiver above the transmitter

For $z>0$, and in the n-th layer $\left(0<z_{n}<h_{n}, 0<n<N+1\right)$ :

$$
\begin{aligned}
H_{n z}^{h}= & \frac{M_{h}}{4 \pi} \sin \phi \int_{0}^{\infty}\left[e^{i u_{n} z_{n}}+R_{U n}^{T E} e^{i u_{n}\left(2 h_{n}-z_{n}\right)}\right] \frac{1-R_{D}^{T E} e^{i 2 u_{0} d_{0}}}{1-R_{U}^{T E} R_{D}^{T E} e^{i 2 u_{0} H}} A_{n} J_{1}\left(k_{r} r\right) k_{r}^{2} d k_{r}, \\
H_{n z}^{h}= & \frac{i M_{h}}{4 \pi} \sin \phi\left\{\int_{0}^{\infty}\left[e^{i u_{n} z_{n}}+R_{U n}^{T E} e^{i u_{n}\left(2 h_{n}-z_{n}\right)}\right] \frac{1-R_{D}^{T E} e^{i 2 u_{0} d_{0}}}{1-R_{U}^{T E} R_{D}^{T E} e^{i 2 u_{0} H}} A_{n} J_{1}\left(k_{r} r\right) u_{n} d k_{r}\right. \\
& +\frac{k_{n}^{2}}{r} \int_{0}^{\infty} \times\left[e^{i u_{n} z_{n}}-R_{U n}^{T M} e^{i u_{n}\left(2 h_{n}-z_{n}\right)}\right] \frac{1+R_{D}^{T M} e^{i 2 u_{0} d_{0}}}{\left.1-R_{U}^{T M} R_{D}^{T M} e^{i 2 u_{0} H} C_{n} J_{1}\left(k_{r} r\right) \frac{1}{u_{0}} d k_{r}\right\},},
\end{aligned}
$$

where $C_{n}$ are obtained with the recursion relationship,

$$
C_{n}=\frac{\alpha_{(n-1)}}{\alpha_{n}} C_{(n-1)} e^{i u_{(n-1) h_{(n-1)}}} \frac{1+R_{U(n-1)}^{T M}}{1+R_{U n}^{T M} e^{i 2 u_{n} h_{n}}},
$$

with $C_{0}=1$, for the layer where the transmitter is situated.

\section{(b) Receiver below the transmitter}

For $z<0$, and in the n-th layer $\left(-d_{n}<z_{n}<0,0<n<M+1\right)$ :

$$
\begin{aligned}
H_{n z}^{h}= & \frac{M_{h}}{4 \pi} \sin \phi \int_{0}^{\infty}\left[e^{-i u_{n} z_{n}}+R_{D n}^{T E} e^{\left.2 d_{n}+z_{n}\right)}\right] \frac{1-R_{D}^{T E} e^{i 2 u_{0} h_{0}}}{1-R_{U}^{T E} R_{D}^{T E} e^{i 2 u_{0} H}} B_{n} J_{1}\left(k_{r} r\right) k_{r}^{2} d k_{r}, \\
H_{n z}^{h}= & \frac{i M_{h}}{4 \pi} \sin \phi\left\{\int_{0}^{\infty}\left[e^{i u_{n} z_{n}}+R_{D n}^{T E} e^{i u_{n}\left(2 d_{n}-z_{n}\right)}\right] \frac{1-R_{U}^{T E} e^{i 2 u_{0} h_{0}}}{1-R_{U}^{T E} R_{D}^{T E} e^{i 2 u_{0} H}} B_{n} J_{1}\left(k_{r} r\right) u_{n} k_{r} d k_{r}\right. \\
& \left.+\frac{k_{n}^{2}}{r} \int_{0}^{\infty} \times\left[e^{-i u_{n} z_{n}}+R_{D n}^{T M} e^{i u_{n}\left(2 d_{n}+z_{n}\right)}\right] \frac{1+R_{U}^{T M} e^{i 2 u_{0} d_{0}}}{1-R_{U}^{T M} R_{D}^{T M} e^{i 2 u_{0} H}} D_{n} J_{1}\left(k_{r} r\right) \frac{1}{u_{0}} d k_{r}\right\},
\end{aligned}
$$

where $D_{n}$ are obtained with the recursion relationship,

$$
D_{n}=\frac{\alpha_{(n-1)}}{\alpha_{n}} D_{(n-1)} e^{i u_{(n-1) d_{(n-1)}}} \frac{1+R_{D(n-1)}^{T M}}{1+R_{D n}^{T M} e^{i 2 u_{n} d_{n}}},
$$

with $D_{0}=1$, for the layer where the transmitter is situated. 
The routine QDAGI of the IMSL package was used to solve numerically these improper integrals. It initially transforms a semiinfinite interval into the finite interval $[0,1]$. Then, it uses a 21 point Gauss-Kronrod rule to estimate the integral and the error. It bisects any interval with an unacceptable error estimate and continues this process until termination.

\section{REFERENCES}

ANDERSON BI \& BARBER TD. 1997. Induction logging - The basics. Schlumberger document SMP-7056. $45 \mathrm{pp}$.

ANDERSON BI, SAFINYA KA \& HABASHY T. 1986. Effects of dipping beds on the response of induction tools. In: Annual Technical Conference and Exhibition. New Orleans, USA. SPE Annals. Expanded Abstracts, 3(1): 29-36.

ANDERSON BI, BORNER S, LÜLING MG \& ROSTHAL R. 1990. Response of 2-MHz LWD resistivity and wireline induction tools tools in dipping beds and laminated formations. In: $31^{\text {st }}$ Ann. Logging Sym., Soc. Prof. Well Log. Analysts, paper A. 24 pp.

ANDERSON BI, BARBER T, BASTIA R, CLAUVAUD JB, COFFIN B, DAS M, HAYDEN R, KLIMENTOS T, MINH CC \& WILLIAMS S. 2008. Triaxial induction $-A$ new angle for an old measurement. Oilfield Review. $84 \mathrm{pp}$.

CARVALHO PR DE. 2000. Respostas eletromagnéticas dos arranjos coplanar e coaxial em poço. D.Sc. Thesis, Department of Geophysics of the Federal University of Pará, Belém, Brazil. 80 pp.

CARVALHO PR DE \& VERMA OP. 1994. Coplanar coil system in EM induction well-logging tool. In: $35^{\text {th }}$ Annual Logging Symposium, Society of Petrophysicists and Well Log Analysts, paper EE. 15 pp.

CARVALHO PR DE \& VERMA OP. 1998. Induction tool with a coplanar coil system. The Log Analyst, 39(6): 48-53.

CARVALHO PR DE \& VERMA OP. 1999. Coplanar coils response in a borehole. In: International Congress of the Brazilian Geophysical Society, 6., Rio de Janeiro, RJ. Proceedings SBGf. v. 2. CD-ROM.

ELLIS DV \& SINGER JM. 2007. Well logging for earth scientists. $2^{\text {nd }}$ ed. Springer. $692 \mathrm{pp}$.

HOWARD AQ JR. 1997. Electromagnetic fields of loop antenna with axis parallel to planar interface. Applied Geophysics, 38: 41-56. Summer: 64-84.
HOWARD AQ JR. 2000. Petrophysics of magnetic dipole fields in an anisotropic earth. IEEE Transactions on Antenas and Propagation, 48(9): 1376-1383.

HOWARD AQ JR \& CHEW WC. 1992. Electromagnetic borehole fields in a layered dipping-bed environment with invasion. Geophysics, 57(3): $451-465$.

KAUFMAN AA \& KELLER GV. 1989. Induction logging. Amsterdam Elsevier Publishers. $600 \mathrm{pp}$.

KRIEGSHÄUSER B, FANINI O, FORGANG S, ITSKOVICH G, RABINOVICH M, TABAROVSKY L \& YU L. 2000. A new multicomponent induction logging tool to resolve anisotropic formations. In: $41^{\text {st }}$ Ann. Logging Sym., Soc. Prof. Well Log. Analysts, paper D. 14 pp.

LU X \& ALUMBAUGH DL. 2001. One-dimensional inversion of threecomponent induction logging in anisotropic media. In: Annual International Meeting, 71. San Antonio. Expanded Abstracts, volume 20, 376380 - Society of Exploration Geophysics.

MORAN JH \& KUNZ KS. 1962. Basic theory of induction logging and application to study of two-coil sondes. Geophysics, 27(6): 829-858.

MORAN JH \& GIANZERO S. 1979. Effects of formation anisotropy on resistivity-logging measurements. Geophysics, 44(7): 1266-1286.

SANTOS WG DOS. 2007. Arranjos coplanar e coaxial nas sondas de perfilagem de poço: ferramentas triaxiais em reservatórios laminados. MSc. Thesis, Department of Geophysics of the Federal University of Pará, Belém, Brazil. 70 pp.

SOUZA NPR \& VERMA OP. 1995. Scale-model response of fracture zones to a coplanar induction tool in a borehole. The Log Analyst, 36(5): 49-57.

WANG H, BARBER T, ROSTHAL R, TABANOU J, ANDERSON BI \& HABASHY T. 2003. Fast and rigorous inversion of triaxial induction logging data to determine formation resistivity anisotropy, bed boundary position, relative dip, and azimuth angles. In: SEG Annual International Meeting, p. 514-517.

WARD SH \& HOHMANN GW. 1988. Electromagnetic theory for geophysical applications. In: NABIGHIAN MN (Ed.). Electromagnetic methods in Applied Geophysics. Theory, 1. Society of Exploration Geophysics. p. 131-311. 


\section{NOTES ABOUT THE AUTHORS}

Paulo Roberto de Carvalho graduated both in Geological Engineering and Mining Engineering from the Universidade Federal de Ouro Preto, Brazil, in 1988 and 1990, respectively. He obtained both his M.Sc. and Ph.D. in Geophysics from the Universidade Federal do Pará, Brazil in 1993 and 2000, respectively. Since 1997, he has been teaching at the Universidade Federal Rural da Amazônia. His interests include electrical and electromagnetic prospecting.

Walleson Gomes dos Santos graduated both in Mathematics and Physics in 2004. He obtained his M.Sc. in Borehole Geophysics from the Universidade Federal do Pará, Brazil in 2007 and is now pursuing a doctorate in electromagnetic methods. His interests include numerical modeling and electromagnetic prospecting.

Cícero Roberto Teixeira Régis got a doctorate in Geophysics from the Universidade Federal do Pará, Brazil, in 1999. He has been teaching Math and Physics, at the secondary and university levels, since 1992. He is member of the Faculty of Geophysics in the UFPA since 2004. His research includes the numerical modeling and inversion of electromagnetic marine geophysical methods. 